\title{
Effect of assist-as-needed robotic gait training on the gait pattern post stroke: a randomized controlled trial
}

\author{
J. F. Alingh ${ }^{1,2^{*}+}$ D, B. M. Fleerkotte $3,4+$, B. E. Groen ${ }^{1,2}$, J. S. Rietman ${ }^{3,4,5}$, V. Weerdesteyn ${ }^{1,2}$, E. H. F. van Asseldonk ${ }^{5}$,
} A. C. H. Geurts ${ }^{1,2+}$ and J. H. Buurke $3,6+$

\begin{abstract}
Background: Regaining gait capacity is an important rehabilitation goal post stroke. Compared to clinically available robotic gait trainers, robots with an assist-as-needed approach and multiple degrees of freedom (AAN $\mathrm{mDOF}_{\text {) }}$ are expected to support motor learning, and might improve the post-stroke gait pattern. However, their benefits compared to conventional gait training have not yet been shown in a randomized controlled trial (RCT). The aim of this two-center, assessor-blinded, RCT was to compare the effect of $\mathrm{AAN}_{\mathrm{mDOF}}$ robotic to conventional training on the gait pattern and functional gait tasks during post-stroke inpatient rehabilitation.
\end{abstract}

Methods: Thirty-four participants with unilateral, supratentorial stroke were enrolled $(<10$ weeks post onset, Functional Ambulation Categories 3-5) and randomly assigned to six weeks of $\mathrm{AAN}_{\mathrm{mDOF}}$ robotic (combination of training in LOPES-II and conventional gait training) or conventional gait training (30 min, 3-5 times a week), focused on pre-defined training goals. Randomization and allocation to training group were carried out by an independent researcher. External mechanical work $\left(\mathrm{W}_{\mathrm{EXT}}\right)$, spatiotemporal gait parameters, gait kinematics related to pre-defined training goals, and functional gait tasks were assessed before training (T0), after training (T1), and at 4-months followup (T2).

Results: Two participants, one in each group, were excluded from analysis because of discontinued participation after T0, leaving 32 participants ( $A A N_{\text {mDOF }}$ robotic $n=17$; conventional $n=15$ ) for intention-to-treat analysis. In both groups, $W_{\text {EXT }}$ had decreased at T1 and had become similar to baseline at T2, while gait speed had increased at both assessments. In both groups, most spatiotemporal gait parameters and functional gait tasks had improved at T1 and T2. Except for step width (T0-T1) and paretic step length (T0-T2), there were no significant group differences at T1 or T2 compared to T0. In participants with a pre-defined goal aimed at foot clearance, paretic knee flexion improved

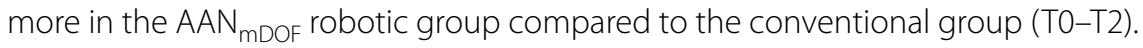

Conclusions: Generally, $A A N_{m D O F}$ robotic training was not superior to conventional training for improving gait pattern in subacute stroke survivors. Both groups improved their mechanical gait efficiency. Yet, $A A N_{m D o F}$ robotic training might be more effective to improve specific post-stroke gait abnormalities such as reduced knee flexion during swing.

\footnotetext{
*Correspondence: j.alingh@maartenskliniek.nl

†J. F. Alingh, B. M. Fleerkotte, A. C. H. Geurts, and J. H. Buurke contributed equally to this work

${ }^{1}$ Sint Maartenskliniek Research, PO Box 9011, 6500 GM Nijmegen, The Netherlands
}

Full list of author information is available at the end of the article

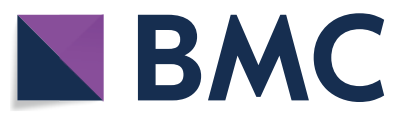

2021. Open Access This article is licensed under a Creative Commons Attribution 4.0 International License, which permits use, sharing, adaptation, distribution and reproduction in any medium or format, as long as you give appropriate credit to the original author(s) and the source, provide a link to the Creative Commons licence, and indicate if changes were made. The images or other third party material in this article are included in the article's Creative Commons licence, unless indicated otherwise in a credit line to the material. If material is not included in the article's Creative Commons licence and your intended use is not permitted by statutory regulation or exceeds the permitted use, you will need to obtain permission directly from the copyright holder. To view a copy of this licence, visit http://creativecommons.org/licenses/by/4.0/. The Creative Commons Public Domain Dedication waiver (http://creativeco mmons.org/publicdomain/zero/1.0/) applies to the data made available in this article, unless otherwise stated in a credit line to the data. 
Trial registration Registry number Netherlands Trial Register (www.trialregister.nl): NTR5060. Registered 13 February 2015.

Keywords: Stroke, Rehabilitation, Gait, Robotics, Quality, External work

\section{Introduction}

Regaining gait capacity is one of the most reported rehabilitation goals post stroke [1-3]. Besides basic gait independence and the ability to adapt gait to environmental demands, rehabilitation is often focused on optimizing the individual gait pattern, particularly in the early phase post stroke. After unilateral supratentorial stroke, the hemiparetic gait pattern is commonly characterized by pes equinovarus during swing and/or loading [4], knee instability during early and/or midstance [5, 6], impaired ankle plantarflexion power during push-off [4], and reduced knee flexion during (pre)swing of the paretic leg [5]. As a consequence, asymmetry in step length [5] and/ or single support time are observed in many patients with post-stroke hemiparesis [7]. In addition, hemiparetic gait is associated with reduced gait speed [8], increased fall risk [9], and limited community ambulation [10]. Hence, improving the post-stroke gait pattern is an important rehabilitation goal.

Robotic gait training has the potential to improve the post-stroke gait pattern [11-17], but its benefits compared to conventional gait training are still under debate [11-18]. Most clinically available robotic gait trainers lack the ability to adjust the robotic actuation based on the user's performance, which may restrain motor learning [18]. In contrast, robotic gait trainers with a so called 'assist-as-needed' (AAN) approach adapt guidance to the user's needs $[19,20]$ and allow support of specific subtasks of the gait cycle [20], thereby promoting active involvement of the user and, thus, motor learning [21-23]. Furthermore, robotic gait trainers with ample degrees of freedom allow a (near) normal gait pattern, in particular with respect to active balance control during walking [21, 24]. In addition, sufficient allowance of movement variability optimizes the amount of error information needed for motor learning [25]. Consequently, robotic gait training with $\mathrm{AAN}$ principles and multiple degrees of freedom $\left(\mathrm{AAN}_{\mathrm{mDOF}}\right.$ ) has the potential to improve gait post stroke. However, no evidence from randomized controlled trials is yet available for its superiority compared to conventional gait training, in particular with regard to the gait pattern, during primary inpatient stroke rehabilitation.

As nearly all kinematic gait deviations and/or spatiotemporal gait abnormalities are translated into irregular movements of the body center of mass of the body $(\mathrm{COM})$, we evaluated the quality of the post-stroke gait pattern based on the COM trajectory. COM movement relative to its surroundings is represented by external mechanical work $\left(\mathrm{W}_{\mathrm{EXT}}\right)$ [26]. In healthy individuals who walk at their preferred speed, COM movements in directions other than the walking direction are typically minimized [27], and $\mathrm{W}_{\mathrm{EXT}}$ is relatively small. Stroke survivors, however, often show compensatory movements in the frontal, sagittal and/or transversal planes while walking, resulting in irregular and enlarged COM trajectories [28] and increased $\mathrm{W}_{\text {EXT }}$ [29], reflecting a reduced quality of the gait pattern. As increased gait speed is generally associated with increased $\mathrm{W}_{\mathrm{EXT}}[30,31]$, interpretation of changes in $\mathrm{W}_{\mathrm{EXT}}$ should be related to changes in gait speed.

The primary aim of the present study was to evaluate whether six weeks $\mathrm{AAN}_{\mathrm{mDOF}}$ robotic gait training would be superior to conventional gait training in terms of $\mathrm{W}_{\mathrm{EXT}}$ in stroke survivors during their inpatient rehabilitation. A secondary aim was to evaluate whether this effect would be retained four months after the intervention. We hypothesized that, given a similar increase in gait speed between groups, the increase in $\mathrm{W}_{\mathrm{EXT}}$ would be smaller following robotic training compared to conventional training one week and four months after the intervention period. A third aim was to evaluate the $\mathrm{AAN}_{\mathrm{mDOF}}$ robotic gait training on spatiotemporal gait parameters, kinematics related to pre-defined training goals, and functional gait tasks.

\section{Methods \\ Participants}

Stroke survivors admitted for inpatient rehabilitation to two rehabilitation centers in the Netherlands (Sint Maartenskliniek, Nijmegen; Roessingh Center for Rehabilitation, Enschede) were assessed for eligibility by their treating rehabilitation physician or physical therapist from October 2015 until June 2019. Inclusion criteria were: (1) adult ( $\geq 18$ years of age) after a first or recurrent unilateral ischemic or hemorrhagic supratentorial stroke (<10 weeks post onset), (2) impairment of one or more prerequisites of gait according to Gage et al. [32]. Exclusion criteria were: (1) inability to walk without support, with or without supervision (Functional Ambulation Category (FAC) 0-2), (2) medical conditions interfering with gait, (3) inability to understand verbal instructions, (4) severe visual problems e.g. hemianopia or visuospatial neglect, (5) no independent ambulation prior to stroke, 
(6) depressed mood assessed with the Hospital Anxiety and Depression Scale (HADS >7), (7) severe lower limb spasticity (at any level) assessed with the Modified Ashworth Scale (MAS $\geq 3$ ), (8) severe lower limb contracture (at any level) determined by a physical examination, (9) body weight $\geq 140 \mathrm{~kg}$, (10) skin problems at any body site where the support harness or straps of the robotic gait trainer were to be fitted, and (11) expected length of stay in rehabilitation center $<6$ weeks. Exclusion criteria 7 to 10 were applied primarily to prevent inappropriate or unsafe fitting of the robotic gait trainer. Individuals who were eligible and willing to participate received study information from the researcher. All participants gave written informed consent before definitive inclusion, in accordance with the Declaration of Helsinki. Demographic and clinical characteristics were collected: sex (male/female), height $(\mathrm{cm})$, hemiparetic side (left/right), use of ankle-foot orthosis (yes/no), lower limb motor impairment (Fugl Meyer Assessment [33]-leg score; 0-34), lower limb strength (Motricity Index [34] - leg score; 0-100), cognition (Montreal Cognitive Assessment (MoCA [35]; 0-30), and communication skills (Utrechts Communicatie Onderzoek (UCO [36]—subscale conversation; $1-5)$.

\section{Study design and randomization}

This study was conducted as a two-center, assessorblinded, randomized controlled, parallel group trial. The study protocol (NL 50748.044.14) was approved by the Medical Ethical Committee Twente (Enschede, the Netherlands) and registered in the Netherlands Trial Register (NTR5060). Figure 1 provides an overview of the study design. Assessments were performed before (T0), within one week after (T1), and four months after (T2) the sixweek intervention period. At each center, all assessments were performed by one assessor who was blinded for group allocation. After completing the T0 assessment, a stratified block randomization with an allocation ratio of 1:1 was used. Participants were stratified by baseline gait speed $(\leq 0.4 \mathrm{~m} / \mathrm{s}$ or $>0.4 \mathrm{~m} / \mathrm{s})$ and allocated to the $\mathrm{AAN}_{\mathrm{mDOF}}$ robotic or conventional gait training groups using random permuted blocks (block sizes two and four) within each strata. An independent researcher generated the random allocation sequence, transferred it to numbered envelopes, and handed the envelope to the participant to inform about the group allocation after completing the $\mathrm{T} 0$ assessment.

\section{Intervention}

Prior to the start of the training an individual training goal was selected by a rehabilitation physician based on clinical examination. The pre-defined training goals were derived from the kinematic aspects of the prerequisites of gait defined by Gage et al. [32] and were operationalized as improving: foot clearance (swing), knee stability (stance), limb loading (stance), or foot prepositioning (swing). The $\mathrm{AAN}_{\mathrm{mDOF}}$ robotic gait training group received three 30-min sessions of individually tailored LOPES II training per week. LOPES II is a treadmill-based $\mathrm{AAN}_{\mathrm{mDOF}}$ robotic gait trainer, combined with a body-weight support system (MOOG BV, Nieuw-Vennep, the Netherlands). LOPES II has eight powered degrees of freedom, actuating pelvic translations in the anterior/posterior and lateral directions, hip flexion/extension, hip adduction/abduction, and knee flexion/extension. Ankle dorsiflexion movements can be supported using toe-lifters or conventional anklefoot orthoses. For a detailed description of the LOPES II see Meuleman et al. [20]. At the start of the training, individually-tailored, minimal levels of body-weight support and general and specific guidance forces were determined at which the participant was just able to match the reference gait trajectories, related to the pre-defined training goal, of the LOPES II. Across the training sessions, the goal was to match the reference gait trajectories of the LOPES II, while gradually reducing the level of body-weight support, reducing the general and specific guidance forces, and increasing the gait speed. Real-time

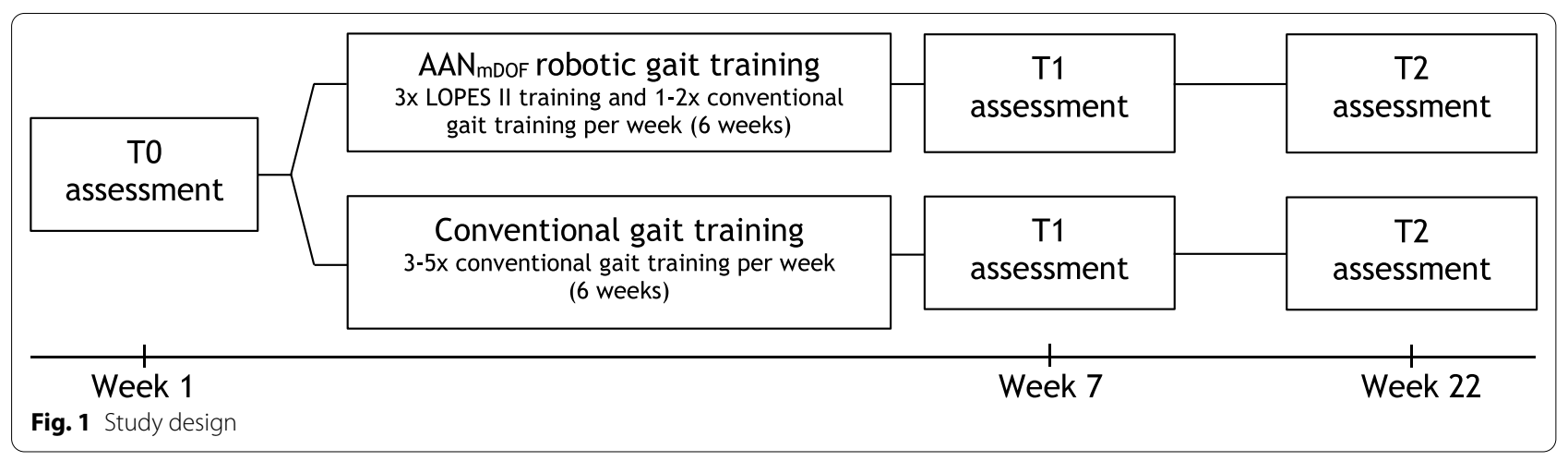


feedback about the participant's gait pattern was provided by the user interface of LOPES II, complemented by verbal feedback from the treating physical therapist. $\mathrm{AAN}_{\mathrm{mDOF}}$ robotic gait training was complemented with a maximum of two 30-min individual gait training sessions per week, according to the latest insights in neurorehabilitation [37]. Thus, when using the term $\mathrm{AAN}_{\mathrm{mDOF}}$ robotic gait training in the remainder of this text, this refers to a combination of robotic gait training in LOPES II and conventional therapy. The training frequency and LOPES II settings were documented in a logbook.

The conventional training group received three to five 30-min individual gait training sessions per week, according to the latest insights in neurorehabilitation [37]. Physical therapists provided verbal feedback about the participant's performance with emphasis on attainment of the individual primary training goal. The training frequency was documented in a logbook. Both the $\mathrm{AAN}_{\mathrm{mDOF}}$ robotic and conventional gait training group could receive group training as part of their regular gait rehabilitation program, in addition to the scheduled individual gait training sessions per week. The training frequency of the group sessions was documented in a logbook. Use of interactive treadmill or other robotic gait trainers was not allowed during the intervention period. After the end of the intervention period (after the T1 assessment), participants were allowed to continue their regular (inpatient or outpatient) rehabilitation program, but these gait training sessions were no longer logged.

\section{Outcomes}

\section{Primary outcome measure}

The primary outcome $\mathrm{W}_{\mathrm{EXT}}$ was determined per stride through analysis of the energy changes at the level of the COM relative to the surroundings [26]. The energy level of the body $\left(\mathrm{E}_{\mathrm{EXT}}\right)$ is determined by the sum of potential and kinetic energy of the COM per stride:

$$
E_{E X T}=\frac{1}{2} M V_{\text {forward }}^{2}+\left(M g H+\frac{1}{2} M V_{\text {vertical }}^{2}\right)+\frac{1}{2} M V_{\text {lateral }}^{2}
$$

where $M$ is the total body mass $(\mathrm{kg}), g$ is gravity $(\mathrm{m} /$ $\left.\mathrm{s}^{2}\right)$, and $H$ and $V$ are the height $(\mathrm{m})$ and velocity in the forward, vertical and lateral direction $(\mathrm{m} / \mathrm{s})$ of the $\mathrm{COM}$ relative to the surrounding. $\mathrm{W}_{\mathrm{EXT}}$ is defined as the sum of the increments of the $\mathrm{E}_{\mathrm{EXT}}$ curve per stride. $\mathrm{W}_{\text {EXT }}$ was normalized for body mass and stride length $(\mathrm{J} / \mathrm{kg} / \mathrm{m})$. As $\mathrm{W}_{\mathrm{EXT}}$ is associated with walking velocity [30,31], $\mathrm{W}_{\mathrm{EXT}}$ is always reported together with gait speed.

\section{Secondary outcome measures}

The following spatiotemporal parameters were calculated using the marker data collected from each trial of the 3D-gait analysis: gait speed $(\mathrm{m} / \mathrm{s})$, step width $(\mathrm{m})$, step length $(\mathrm{m})$, and single-support time (\% gait cycle). Symmetry ratios were calculated for step length and singlesupport time, and expressed as the absolute difference from 0.5 (perfect symmetry), according to the following equation:

$$
\text { Symmetryratio }=\left|0.5-\frac{\text { Parameter }_{\text {pareticleg }}}{\text { Parameter }_{\text {non-pareticleg }}+\text { Parameter }_{\text {pareticleg }}}\right|
$$

\section{Procedure}

During each assessment a 3D-gait analysis was performed. Reflective markers $(n=39)$ were attached to the participant according to the Plug-In-Gait Full Body model (Plug-In-Gait, Vicon Motion Systems Ltd, Oxford, UK). Marker positions were recorded by infrared cameras $\left(f_{s}=100 \mathrm{~Hz}\right.$; Vicon $\mathrm{mX}$ 1.7.1, Oxford Metrics, UK). Participants were instructed to walk at their self-selected speed along a straight 6-m walkway. Participants wore their own shoes and were allowed to use an ankle-foot orthosis if necessary, which could vary between assessments as a consequence of motor recovery. Use of other walking aids was not allowed. At least 15 strides were collected during each assessment. Data was analyzed using custom written software (MATLAB, Mathworks Inc, Natick, MA, USA). Initial contact and foot-off were determined with the velocity-based algorithm as described by Zeni et al. [38].
In addition, the following functional gait tasks and clinical leg motor scores were recorded during each assessment: 6-Minute Walk Test [39], 10-Meter Walk Test [40], Timed Up and Go Test [41], Functional Gait Assessment [42], Fugl Meyer Assessment [33] —leg score, and Motricity Index [34] - leg score. Participants were allowed to use an ankle-foot orthosis and/or a walking aid during the functional gait tasks when necessary.

\section{Individual training goals}

To evaluate the training effects on the pre-defined training goals, Vicon Plug-In-Gait model and software were used to calculate the individual gait kinematics per stride. Foot clearance, knee stability in stance (reduction in knee extension thrust), limb loading and foot prepositioning were evaluated by maximal knee flexion of the paretic leg during early swing, the difference in maximal knee 
extension velocity between the paretic and non-paretic leg during single-support phase, single-support time symmetry, and minimal knee flexion of the paretic leg during terminal swing, respectively.

\section{Power calculation}

Power analysis performed using STATA version 10.1 showed that a sample size of 50 participants $(\alpha=0.05$, $\beta=0.10$, including $10 \%$ drop-out) was sufficient to demonstrate a group difference in $\mathrm{W}_{\mathrm{EXT}}$ of $0.13 \mathrm{~J} / \mathrm{kg} / \mathrm{m}$ after the intervention [43].

\section{Statistical analysis}

All statistical analyses were performed using SPSS statistics version 19 (IBM SPSS Statistics, Chicago, USA). Baseline characteristics were compared between groups using independent $\mathrm{t}$-tests or Mann-Whitney $\mathrm{U}$ tests for continuous variables, and chi-square tests for categorical variables. $\mathrm{W}_{\mathrm{EXT}}$, spatiotemporal parameters, and gait kinematics were averaged per individual over all strides per assessment (T0-T2). Effects of the intervention at T1 and T2 on primary and secondary outcomes were separately analyzed, according to an intention-totreat' principle, using linear mixed model for repeated measures with a fixed effect for Group $\left(\mathrm{AAN}_{\mathrm{mDOF}}\right.$ robotic vs conventional) and Time (T0 vs $\mathrm{T} 1$, or T0 vs T2). All linear mixed models used a restricted maximum likelihood estimation to obtain the results, an unstructured covariance matrix, and Šidák adjustment for multiple testing. Effects of the intervention on the pre-defined training goals were analyzed per subgroup of participants with the same pre-defined training goal $(\mathrm{n} \geq 10)$, according to an 'per-protocol' analysis, using non-parametric Mann-Whitney U tests on difference scores for each outcome ( $\mathrm{T} 0$ vs $\mathrm{T} 1$, or T0 vs T2). The significance level was set at $\mathrm{p}<0.05$ for all tests.

\section{Results}

The participants' flow is presented in Fig. 2. Recruitment started in October 2015 and was stopped in June 2019 due to end of funding. Thirty-four individuals were randomly assigned to the $\mathrm{AAN}_{\mathrm{mDOF}}$ robotic $(\mathrm{n}=18$; gait speed $<0.4 \mathrm{~m} / \mathrm{s}, \mathrm{n}=7$ ) or conventional gait training group $(n=16$; gait speed $<0.4 \mathrm{~m} / \mathrm{s}, \mathrm{n}=6)$. Two participants, one in each group, discontinued participation directly after T0, because they expected the study protocol to be too physically demanding. Hence 32 participant were included in the intention-totreat analysis $\left(\mathrm{AAN}_{\mathrm{mDOF}}\right.$ robotic $\mathrm{n}=17$; conventional $\mathrm{n}=15$ ). One participant discontinued the robotic gait training, because the study protocol was too physically demanding. Another four subjects $\left(\mathrm{AAN}_{\mathrm{mDOF}}\right.$ robotic $\mathrm{n}=3$; conventional $\mathrm{n}=1$ ) were lost to follow-up after the post-intervention assessment, because of time requirements $(n=2)$ or medical reasons unrelated to the study $(\mathrm{n}=2)$. Baseline demographic and clinical characteristics, and individual training goals did not differ between groups (see Table 1).

\section{Details of interventions and adverse effects}

In the robotic training group, one participant discontinued training after 2 sessions, whereas the other participants received a median of 15 (interquartile range (IQR): 13.8-15.3) individual robotic gait training sessions. In accordance with the training protocol, a reduction in average body weight support (week 1: $8.3 \pm 5.6 \%$; week 6: $6.7 \pm 3.5 \%$ ), general guidance force (week 1 : $61.1 \pm 22.0 \%$; week $6: 22.2 \pm 25.6 \%$ ), and specific guidance force (week 1: $42.8 \pm 21.8 \%$; week 6: $25.0 \pm 23.2 \%$ ) was applied, while average gait speed was increased (week 1: $1.60 \pm 0.51 \mathrm{~km} / \mathrm{h}$; week $6: 2.40 \pm 0.62 \mathrm{~km} / \mathrm{h}$ ) across the robotic training sessions. In addition to the robotic gait training, participants in this group received a median of 11 (IQR: 7.5-12.0) individual and 6 (IQR: 4.8-10.5) group sessions of conventional gait training, resulting in a total median number of 32 (IQR: 26.0-37.8) training sessions during the intervention period. The conventional training group received a median of 18 (IQR: 14.5-22.0) individual and 9 (IQR: 7.5-12.0) group sessions of conventional gait training, resulting in a total median number of 27 (IQR: 22.0-34.0) training sessions during the intervention period. One participant experienced a fall with wheelchair, outside the study context, but was able to continue conventional gait training after one week of rest. No additional adverse events were reported.

\section{External mechanical work and gait speed}

Group results of $\mathrm{W}_{\mathrm{EXT}}$ and gait speed are summarized in Table 2. The corresponding test statistics are reported in Additional file 1. Irrespective of group allocation (Group $\times$ Time interactions, $\mathrm{p} \geq 0.438), \quad \mathrm{W}_{\text {EXT }}$ significantly decreased from $\mathrm{T} 0$ to $\mathrm{T} 1$ (mean difference $=-0.09 \mathrm{~J} /$ $\mathrm{kg} / \mathrm{m}$; $95 \%$ CI -0.17 to $-0.01, \mathrm{p}=0.039$ ), while gait speed significantly increased from $\mathrm{T} 0$ to $\mathrm{T} 1$ (mean difference $=0.15 \mathrm{~m} / \mathrm{s} ; 95 \%$ CI 0.08-0.22, $\mathrm{p}<0.001$ ) (see Fig. 3). Figure 4 shows that 21 out of 31 participants who completed both assessments had lower $\mathrm{W}_{\mathrm{EXT}}$ at T1. Seventeen of them (81\%) showed a concurrent increase in gait speed, whereas four participants (19\%) showed a concurrent decrease in gait speed. Of the 10 participants with increased $\mathrm{W}_{\mathrm{EXT}}$ at $\mathrm{T} 1$, eight $(80 \%)$ showed a concurrent increase and two $(20 \%)$ a decrease in gait speed. 


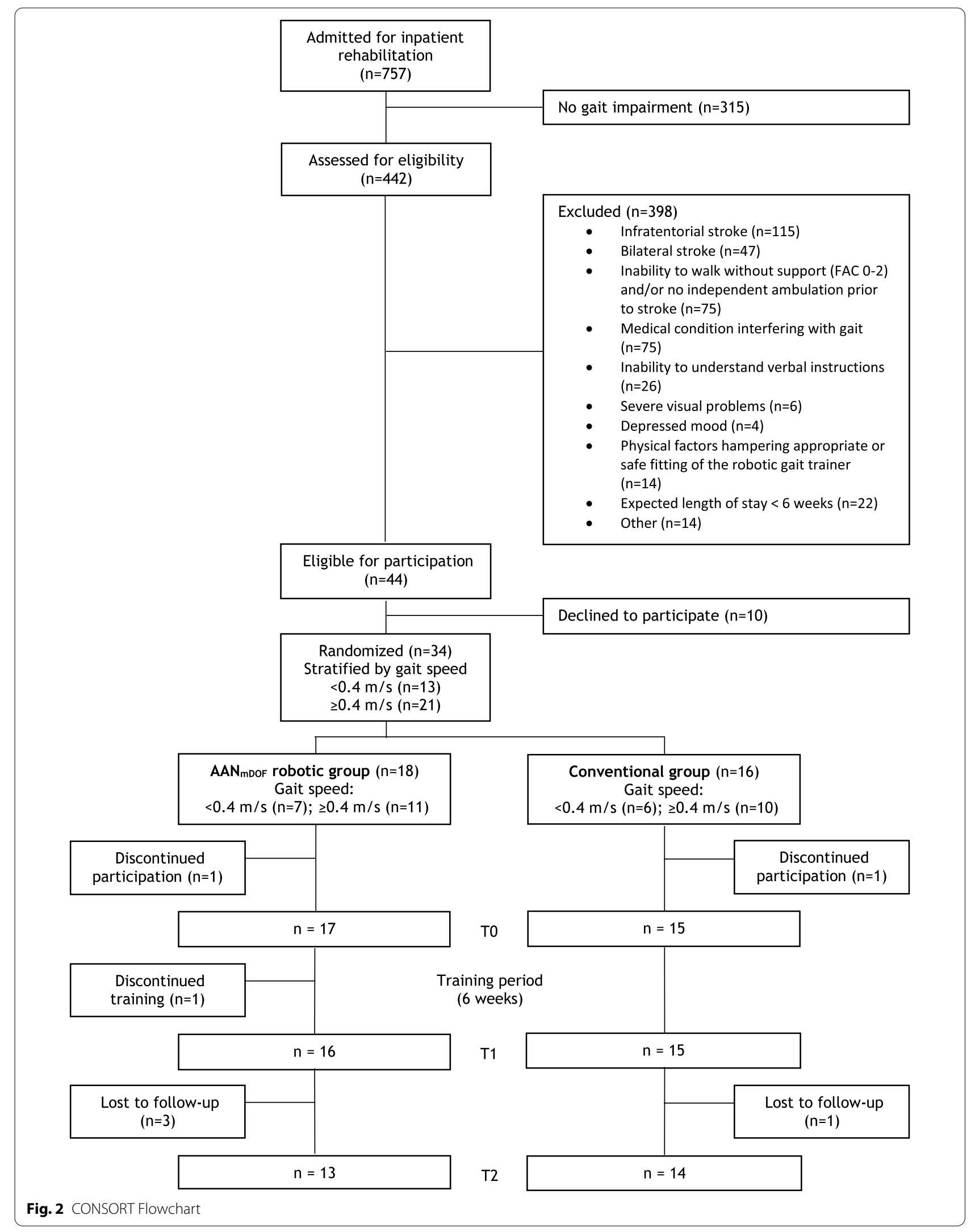


Table 1 Baseline demographic, clinical characteristics, and individual training goals for the $A A N_{\text {mDOF }}$ robotic and conventional gait training groups (mean \pm SD or number)

\begin{tabular}{|c|c|c|}
\hline & $\begin{array}{l}\text { AAN }_{\text {mDoF }} \text { robotic } \\
n=17\end{array}$ & $\begin{array}{l}\text { Conventional } \\
n=15\end{array}$ \\
\hline Sex, male/female (n) & $10 / 7$ & $10 / 5$ \\
\hline Age (years) & $60.6 \pm 9.3$ & $56.8 \pm 9.8$ \\
\hline Height (cm) & $177.4 \pm 7.6$ & $177.7 \pm 7.5$ \\
\hline Weight (kg) & $80.8 \pm 16.0$ & $79.3 \pm 14.3$ \\
\hline $\begin{array}{l}\text { Type of stroke, ischemic/haemor- } \\
\text { rhagic (n) }\end{array}$ & $13 / 4$ & $11 / 4$ \\
\hline Time since stroke (wks) & $5.4 \pm 1.8$ & $5.9 \pm 2.1$ \\
\hline Hemiparetic side, left/right (n) & $7 / 10$ & $7 / 8$ \\
\hline Use of ankle-foot orthosis (n) & 8 & 6 \\
\hline \multicolumn{3}{|l|}{ FAC score $(n)$} \\
\hline 3 & 10 & 6 \\
\hline 4 & 6 & 8 \\
\hline 5 & 1 & 1 \\
\hline Fugl Meyer Assessment—leg score & $24.2 \pm 4.6$ & $23.4 \pm 6.8$ \\
\hline Motricity Index-leg score & $63.9 \pm 17.0$ & $62.5 \pm 26.4$ \\
\hline HADS—subscale depression & $1.8 \pm 1.5$ & $1.2 \pm 0.9$ \\
\hline MoCA & $24.1 \pm 4.2$ & $23.4 \pm 4.1$ \\
\hline \multicolumn{3}{|l|}{ UCO_subscale conversation (n) } \\
\hline 4 & 0 & 2 \\
\hline 5 & 17 & 13 \\
\hline \multicolumn{3}{|l|}{ Individual training goal (n) } \\
\hline Foot clearance & 6 & 6 \\
\hline Knee stability & 6 & 7 \\
\hline Limb loading & 4 & 2 \\
\hline Foot prepositioning & 1 & 0 \\
\hline
\end{tabular}

FAC score Functional Ambulation Category (range 0-5), Fugl Meyer Assessmentleg score (range 0-34), Motricity Index leg score (range 0-100), HADS Hospital Anxiety and Depression Scale-subscale depression (range 0-21), MoCA Montreal Cognitive Assessment (range 0-30), UCO Utrechts Communicatie Onderzoeksubscale conversation (range 1-5)

Between T0 and T2, $\mathrm{W}_{\text {EXT }}$ did not significantly differ $(\mathrm{p}=0.263)$, while gait speed significantly increased in the same time period (mean difference $=0.26 \mathrm{~m} / \mathrm{s} ; 95 \% \mathrm{CI}$ 0.18-0.34; $\mathrm{p}<0.001$ ) (see Fig. 3). These Time effects did not differ between groups (Group $\times$ Time interactions, $\mathrm{p} \geq 0.152$ ).

\section{Secondary outcomes}

Paretic and non-paretic step length, paretic single-support time, step length and single-support time symmetry, and all functional gait tasks and clinical scores significantly improved from $\mathrm{T} 0$ to $\mathrm{T} 1$ (improvements ranging from 7.4 to $37.9 \% ; \mathrm{p}<0.049$ ) and $\mathrm{T} 0$ to $\mathrm{T} 2$ (improvements ranging from $14.5 \%$ to $67.6 \%$; $<<0.019$ ) (see Table 2 and Additional file 1). In addition, non-paretic single-support time improved from T0 to T1 $(\mathrm{p}=0.005)$, and did not differ between T0 and T2 $(\mathrm{p}=0.075)$. Most Time effects were similar for both groups from T0 to T1 (Group $\times$ Time interactions $\mathrm{p} \geq 0.106$ ), as well as from T0 to T2 (Group $\times$ Time interactions $(\mathrm{p} \geq 0.063)$. From T0 to T1, the only significant difference between group was found for step width, which remained constant following robotic gait training, whereas it increased by $2 \mathrm{~cm}$ after conventional training (Group $\times$ Time interaction $\mathrm{p}=0.018$ ). Step width was similar for both groups between T0 to T2 (Group $\times$ Time interaction $\mathrm{p}=0.055)$. Furthermore, from T0 to T2, the increase in paretic step length was larger following robotic gait training $(16 \mathrm{~cm})$ compared to conventional gait training $(6 \mathrm{~cm}$; Group $\times$ Time interaction $\mathrm{p}=0.027)$. There were no main effects of Group for any outcome from T0 to T1 ( $\mathrm{p} \geq 0.152)$ or from T0 to T2 ( $\mathrm{p} \geq 0.201)$.

\section{Individual training goals}

Participants with a pre-defined training goal aimed at foot clearance $(n=12)$ did not show a significant difference in the change in peak knee flexion between the robotic and conventional training group from $\mathrm{T} 0$ to $\mathrm{T} 1$ $(\mathrm{p}=0.055)$, but this parameter reached significance in favor of the robotic training group when comparing T0 with T2 ( $\mathrm{p}=0.016$, effect size $r=0.55)$ (see Table 3 and Additional file 2). Participants with a pre-defined training goal aimed at knee stability $(\mathrm{n}=13)$ did not show significant differences in the change in maximal knee extension velocity of the paretic relative to the non-paretic leg between groups for either time interval (T0 vs T1, $\mathrm{p}=0.570$; T0 vs $\mathrm{T} 2, \mathrm{p}=0.796$ ). Six participants had a primary training goal aimed at improving limb loading and one participant at improving foot prepositioning. These subgroups were considered too small to allow statistical sub-analysis.

\section{Discussion}

Our hypothesis that, in the subacute phase after stroke, six weeks of $\mathrm{AAN}_{\mathrm{mDOF}}$ robotic gait training would be superior to conventional gait training in terms of $\mathrm{W}_{\mathrm{EXT}}$ (as a generic measure of the quality of the gait pattern) was not corroborated by the results of this study. Both the $\mathrm{AAN}_{\mathrm{mDOF}}$ robotic and conventional gait training groups showed equally reduced $\mathrm{W}_{\mathrm{EXT}}$ one week after the intervention period, combined with similarly increased gait speed. At four months follow-up, there was a further and similar increase in gait speed in both groups, while $\mathrm{W}_{\text {EXT }}$ returned to baseline values. In addition, compared to baseline, most spatiotemporal parameters, all functional gait tasks and all clinical scores had similarly improved in both groups one week after the intervention and at follow-up. The $\mathrm{AAN}_{\mathrm{mDOF}}$ robotic gait training 
Table 2 Means ( \pm SDs) of mechanical work, spatiotemporal gait parameters, functional gait tasks, and clinical scores for the $A A N_{m D O F}$ robotic and conventional gait training groups, before (T0), immediately after (T1), and four months after (T2) the six-week intervention period

\begin{tabular}{|c|c|c|c|c|c|c|}
\hline & \multicolumn{3}{|c|}{ AAN $_{\text {mDOF }}$ robotic } & \multicolumn{3}{|c|}{ Conventional } \\
\hline & $\begin{array}{l}\text { T0 } \\
n=17\end{array}$ & $\begin{array}{l}T 1 \\
n=16\end{array}$ & $\begin{array}{l}T 2 \\
n=13\end{array}$ & $\begin{array}{l}\text { T0 } \\
n=15\end{array}$ & $\begin{array}{l}T 1 \\
n=15\end{array}$ & $\begin{array}{l}T 2 \\
n=14\end{array}$ \\
\hline \multicolumn{7}{|l|}{ Mechanical work } \\
\hline$W_{\text {EXT }}(\mathrm{J} / \mathrm{kg} / \mathrm{m})^{*}$ & $0.61 \pm 0.25$ & $0.52 \pm 0.18$ & $0.69 \pm 0.26$ & $0.63 \pm 0.23$ & $0.53 \pm 0.20$ & $0.70 \pm 0.29$ \\
\hline \multicolumn{7}{|l|}{ Spatiotemporal parameters } \\
\hline Gait speed $(\mathrm{m} / \mathrm{s}) * * *$ & $0.47 \pm 0.36$ & $0.67 \pm 0.29$ & $0.81 \pm 0.24$ & $0.62 \pm 0.36$ & $0.75 \pm 0.38$ & $0.81 \pm 0.32$ \\
\hline Step width $(m)^{* \dagger}$ & $0.15 \pm 0.04$ & $0.15 \pm 0.05$ & $0.14 \pm 0.05$ & $0.16 \pm 0.06$ & $0.18 \pm 0.06$ & $0.17 \pm 0.06$ \\
\hline \multicolumn{7}{|l|}{ Step length } \\
\hline Paretic $(m) * * * \neq$ & $0.35 \pm 0.15$ & $0.46 \pm 0.11$ & $0.51 \pm 0.10$ & $0.43 \pm 0.13$ & $0.48 \pm 0.13$ & $0.49 \pm 0.12$ \\
\hline Non-paretic $(m) * * *$ & $0.33 \pm 0.17$ & $0.44 \pm 0.14$ & $0.50 \pm 0.09$ & $0.39 \pm 0.20$ & $0.46 \pm 0.19$ & $0.50 \pm 0.15$ \\
\hline Symmetry ratio *** & $0.04 \pm 0.05$ & $0.04 \pm 0.03$ & $0.03 \pm 0.01$ & $0.07 \pm 0.10$ & $0.04 \pm 0.06$ & $0.03 \pm 0.03$ \\
\hline \multicolumn{7}{|l|}{ Single-support time } \\
\hline Paretic (\% gait cycle) ${ }^{* * *}$ & $0.28 \pm 0.08$ & $0.33 \pm 0.06$ & $0.34 \pm 0.05$ & $0.31 \pm 0.07$ & $0.33 \pm 0.05$ & $0.33 \pm 0.04$ \\
\hline Non-paretic (\% gait cycle) ${ }^{*}$ & $0.32 \pm 0.06$ & $0.36 \pm 0.05$ & $0.36 \pm 0.04$ & $0.36 \pm 0.05$ & $0.37 \pm 0.05$ & $0.36 \pm 0.04$ \\
\hline Symmetry ratio *** & $0.05 \pm 0.05$ & $0.03 \pm 0.04$ & $0.03 \pm 0.03$ & $0.04 \pm 0.03$ & $0.04 \pm 0.03$ & $0.03 \pm 0.03$ \\
\hline \multicolumn{7}{|l|}{ Functional gait tasks } \\
\hline 10-Meter Walk Test $(\mathrm{m} / \mathrm{s}) * * *$ & $0.61 \pm 0.35$ & $0.86 \pm 0.38$ & $1.07 \pm 0.27$ & $0.76 \pm 0.38$ & $0.95 \pm 0.38$ & $1.07 \pm 0.40$ \\
\hline 6-Minute Walk Test $(m) * * *$ & $220 \pm 149$ & $301 \pm 163$ & $398 \pm 119$ & $247 \pm 130$ & $343 \pm 147$ & $383 \pm 138$ \\
\hline Functional Gait Assessment *** & $14.7 \pm 5.7$ & $19.5 \pm 4.8$ & $23.6 \pm 5.4$ & $15.9 \pm 5.8$ & $21.7 \pm 5.2$ & $21.7 \pm 5.3$ \\
\hline Timed Up and Go test $(s) * * *$ & $23.1 \pm 15.0$ & $17.0 \pm 14.4$ & $11.4 \pm 6.1$ & $19.5 \pm 11.8$ & $14.1 \pm 7.6$ & $12.4 \pm 6.4$ \\
\hline \multicolumn{7}{|l|}{ Clinical scores } \\
\hline Fugl Meyer Assessment - leg score $* * *$ & $24.2 \pm 4.6$ & $26.4 \pm 5.0$ & $28.9 \pm 4.1$ & $23.4 \pm 6.8$ & $28.9 \pm 4.1$ & $26.8 \pm 5.5$ \\
\hline Motricity Index - leg score ${ }^{* *}$ & $63.9 \pm 17.0$ & $77.0 \pm 13.7$ & $86.2 \pm 13.2$ & $62.5 \pm 26.4$ & $71.8 \pm 24.6$ & $72.5 \pm 22.2$ \\
\hline
\end{tabular}

Functional Gait Assessment: range 0-30; Fugl Meyer Assessment - leg score: range 0-34; Motricity Index - leg score: range 0-100.

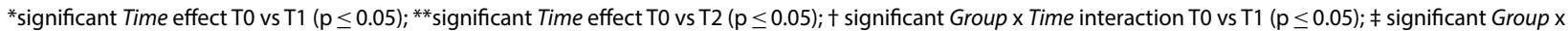
Time interaction T0 vs T2 ( $p$-value $\leq 0.05$ );

group showed no difference in step width one week after the intervention, in contrast to a slight increase in the conventional training group. In addition, at follow-up, paretic step length had increased only in the $\mathrm{AAN}_{\mathrm{mDOF}}$ robotic gait training group. Furthermore, of all patients with a predefined goal aimed at foot clearance, only those who received $\mathrm{AAN}_{\mathrm{mDOF}}$ robotic gait training were able to improve their maximal knee flexion after the intervention. No such subgroup differences were observed for patients with other predefined goals such as knee stability or limb loading.

Overall, our findings do not indicate a clear superior effect of $\mathrm{AAN}_{\mathrm{mDOF}}$ robotic gait training compared to conventional gait training during primary inpatient stroke rehabilitation. Although the conventional gait training group showed a potentially undesirable increase in step width directly after the intervention period, the change was very small $(2 \mathrm{~cm})$ and step width at followup remained similar in both groups. Additionally, the $\mathrm{AAN}_{\mathrm{mDOF}}$ robotic gait training group had increased their paretic step length at follow-up more than the conventional training group, but this effect was related to a shorter paretic step length at baseline in the robotic group. Indeed, both groups reached almost perfect symmetry at follow-up. Consequently, the clinical relevance of these findings is questionable. Hence, the data suggest that people after stroke recover in terms of motor impairments (clinical scores) and motor capacities ( $\mathrm{W}_{\mathrm{EXT}}$, gait speed, symmetry, and functional gait tasks) independent of the type of gait training. Our findings are in line with previous studies reporting beneficial effects of $\mathrm{AAN}_{\mathrm{mDOF}}$ robotic gait training (not complemented with conventional gait training) on the over ground gait pattern and on clinical outcomes in chronic stroke survivors [44-46]. Furthermore, such AAN $\mathrm{mDOF}_{\mathrm{m}}$ robotic gait training combined with functional electrical stimulation was not found to be superior to therapist-assisted body-weight supported treadmill training in a small group of stroke survivors [45]. Hence, the findings of our randomized controlled trial add up to the current evidence that the effectiveness of $\mathrm{AAN}_{\mathrm{mDOF}}$ robotic gait training is limited, 

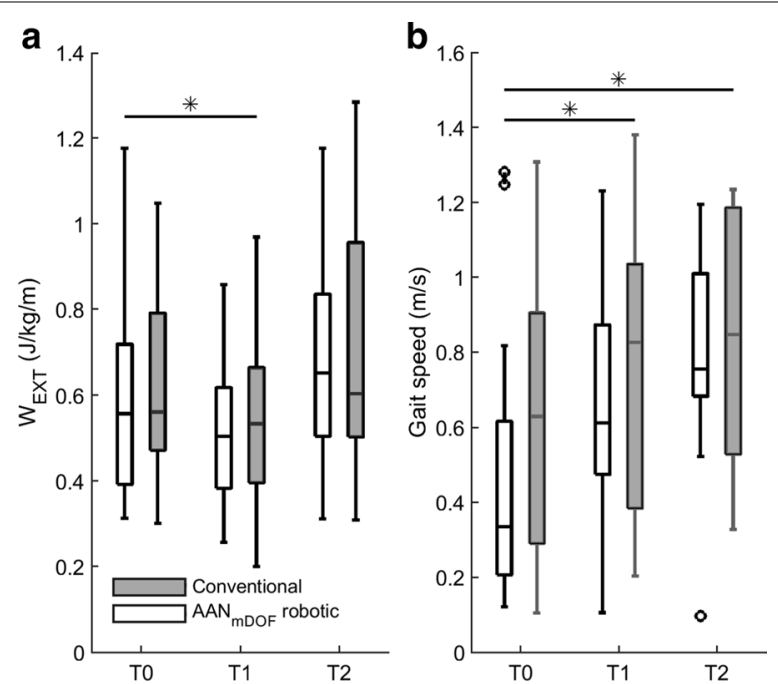

Fig. 3 Course of change in $\mathbf{a}$ external mechanical work and $\mathbf{b}$ gait speed across assessments (T0-T2) in the AAN $\mathrm{mDOF}_{\text {mo }}$ robotic and conventional gait training groups. Each box represents the median, and upper and lower quartiles of the variable, with whiskers extended to the extreme values. Outliers are represented by markers. * significant Time effect $(p<0.05)$

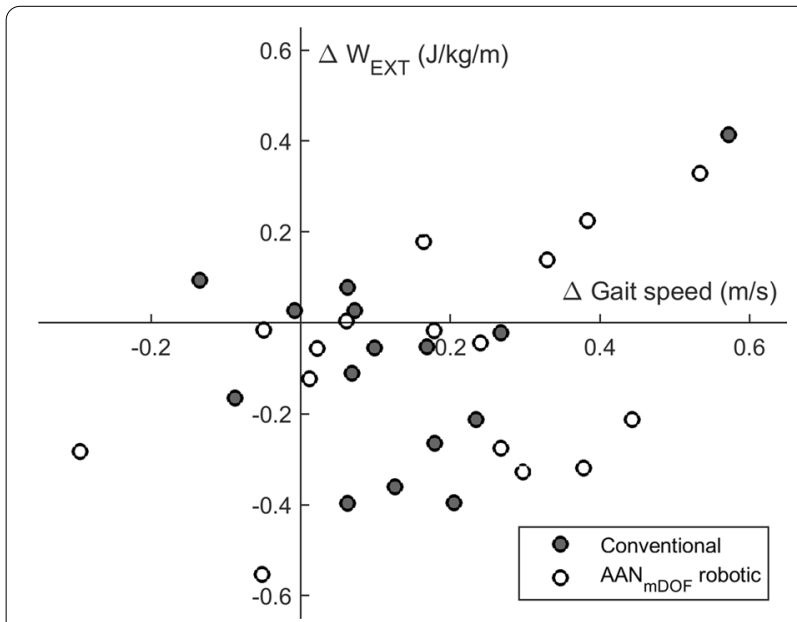

Fig. 4 Individual change in gait speed plotted against the individual change in external mechanical work from T0 to T1, for individuals in the $\mathrm{AAN}_{\mathrm{mDOF}}$ robotic and conventional gait training groups. Only data of individuals who completed both assessments at T0 and T1 are shown $(n=31)$. Positive change indicates an increased value of the variable at T1 relative to T0. Preferably, participants would be in the right lower quadrant (increased gait speed / decreased external work) or lower part of the right upper quadrant (increased gait speed / slightly increased external work)

but that $\mathrm{AAN}_{\mathrm{mDOF}}$ robotic gait training might be used as an alternative for conventional gait training.

One week after the intervention, an increase in gait speed and concurrent decrease in $\mathrm{W}_{\mathrm{EXT}}$ was observed in both groups. In contrast, previous studies have shown that faster gait speed is typically associated with increased levels of $\mathrm{W}_{\mathrm{EXT}}[26,30,31]$. In line, eight of our participants had increased their gait speed and increased their $\mathrm{W}_{\mathrm{EXT}}$ accordingly (see Fig. 4). However, most of our participants $(\mathrm{n}=17)$ showed an increased gait speed and a concurrent decrease in $\mathrm{W}_{\mathrm{EXT}}$. This observed decrease in $\mathrm{W}_{\mathrm{EXT}}$ while walking at a faster speed can be explained by reduced COM movements relative to the surroundings, suggesting that participants reduced their (compensatory) movements in the planes perpendicular to the walking direction. Taken together, these results indicate a more mechanically efficient, and better qualitative gait pattern one week after the intervention in both groups, which is supported by concurrent improvements in gait symmetry in both groups. Interestingly, at follow-up, the gait speed had further increased in both groups, however, now combined with a concurrent increase in $\mathrm{W}_{\mathrm{EXT}}$ to baseline values. This suggests a further increase in functional gait capacity with a stabilization of mechanical efficiency and quality of the gait pattern in both groups four months after the intervention.

Although the analysis of individual training goals demonstrated mixed results, of all participants with a predefined goal aimed at improving foot clearance, only those who received robotic gait training had increased their peak knee flexion during swing at follow-up $(+66 \%)$, whereas peak knee flexion had decreased at follow-up in those who received conventional gait training $(-19 \%)$ (see Table 3). Individuals in the conventional gait training group may have relied more on compensatory pelvic hike and hip abduction ('circumduction') to ensure foot clearance [5]. Although the effect size of this subgroup difference seems to be fairly large, the statistics are based on a small group size and, thus, should be interpreted with caution. It might be that individuals in the $\mathrm{AAN}_{\mathrm{mDOF}}$ robotic gait training group benefited from appropriate proprioceptive information through continuous adaption of knee joint guidance from the $\mathrm{AAN}_{\mathrm{mDOF}}$ robot. Therefore, $\mathrm{AAN}_{\mathrm{mDOF}}$ robotic gait training that can support specific subtasks of the gait cycle seems to have the possibility to promote gait kinematics, but further research with larger group sizes is needed to determine its effect on all prerequisites of gait.

A limitation of the present study is that the generalizability of our results is limited to people suffering from primary or recurrent unilateral supratentorial stroke with independent ambulation prior to their stroke, a minimal level of independent ambulation after their stroke, and without relevant comorbidities. As a consequence, merely $7.5 \%$ of the individuals assessed for eligibility were eventually randomized to one of the training groups. Because participants had to be able 
Table 3 Medians (ranges) of gait kinematics related to individual pre-defined training goals for the $A A N_{\text {mDof }}$ robotic and conventional gait training groups before (T0), immediately after (T1), and four months after (T2) the intervention period

\begin{tabular}{|c|c|c|c|c|c|c|}
\hline & \multicolumn{3}{|c|}{$\mathrm{AAN}_{\text {mDOF }}$ robotic } & \multicolumn{3}{|l|}{ Conventional } \\
\hline & T0 & $\mathrm{T} 1$ & $\mathrm{~T} 2$ & T0 & $\mathrm{T} 1$ & $\mathrm{~T} 2$ \\
\hline Foot clearance & $n=6$ & $n=6$ & $n=6$ & $n=6$ & $n=6$ & $n=6$ \\
\hline Peak knee flexion $\left({ }^{\circ}\right)^{* *}$ & $\begin{array}{l}31.6 \\
(11.4-54.3)\end{array}$ & $\begin{array}{l}47.9 \\
(16.3-60.0)\end{array}$ & $\begin{array}{l}52.4 \\
(16.9-59.5)\end{array}$ & $\begin{array}{l}42.1 \\
(29.5-56.6)\end{array}$ & $\begin{array}{l}36.5 \\
(22.4-64.1)\end{array}$ & $\begin{array}{l}33.9 \\
(20.7-53.5)\end{array}$ \\
\hline Knee stability & $n=6$ & $n=5$ & $n=3$ & $n=7$ & $n=7$ & $n=6$ \\
\hline $\begin{array}{l}\text { Difference in paretic vs non-paretic } \\
\text { maximum knee extension velocity } \\
(\% / s)\end{array}$ & $\begin{array}{l}-13.7 \\
(-76.2-27.5)\end{array}$ & $\begin{array}{l}-9.0 \\
(-22.9-37.4)\end{array}$ & $\begin{array}{l}-20.9 \\
(-67.3-32.7)\end{array}$ & $\begin{array}{l}-25.6 \\
(-100.6-41.3)\end{array}$ & $\begin{array}{l}-34.6 \\
(-79.3-67.1)\end{array}$ & $\begin{array}{l}-7.5 \\
(-55.3-53.0)\end{array}$ \\
\hline Limb loading & $n=4$ & $\mathrm{n}=4$ & $n=3$ & $n=2$ & $\mathrm{n}=2$ & $n=2$ \\
\hline Single-support time symmetry ratio & $\begin{array}{l}0.02 \\
(0.01-0.15)\end{array}$ & $\begin{array}{l}0.01 \\
(0-0.14)\end{array}$ & $\begin{array}{l}0.01 \\
(0-0.13)\end{array}$ & $\begin{array}{l}0.04 \\
(0.04-0.04)\end{array}$ & $\begin{array}{l}0.02 \\
(0-0.03)\end{array}$ & $\begin{array}{l}0.02 \\
(0-0.04)\end{array}$ \\
\hline
\end{tabular}

**Significant between group difference T0 vs T2 $(p \leq 0.05)$. The individual pre-defined training goal foot prepositioning was excluded from analysis because $n=1$

to perform the gait analysis independently, individuals with poor (dependent) ambulatory capacity were excluded. As this latter group may typically profit from mechanically assisted gait training [47], it is still relevant to investigate the effect of $\mathrm{AAN}_{\mathrm{mDOF}}$ robotic gait training in those with more severely affected gait capacity after stroke. A second limitation is that the study may lack sufficient power, as the number of included participants was smaller than the calculated sample size. Nevertheless, it should be noted that the original power calculation was based on a Beta of $10 \%$. Using a Beta of $20 \%$ would have required 36 participants. Given the current sample size of 34 participants and the absence of any trend in the Group $\times$ Time interaction effects, we assume that the chance of false-negative study results is very small. A third limitation is that, with regard to the gait training, the $\mathrm{AAN}_{\mathrm{mDOF}}$ robotic training group ultimately received $19 \%$ more training sessions than the conventional training group. This difference in training intensity might have worked in favor of the robotic group, but the results did not show any indication of such an effect. Lastly, the calculation of $\mathrm{W}_{\mathrm{EXT}}$ was based on the COM movements derived from the gait kinematics instead of integrating ground reaction forces [48]. As it was difficult for several participants to successfully hit the force plate during gait analysis, ground reaction forces could not be recorded in a sufficient number of steps to be analyzed properly. Although the use of COM movements derived from kinematics implies multiple assumptions about anthropometry, rigidity of body segments, and correct marker placement, this method still appears to be valid for calculating $\mathrm{W}_{\mathrm{EXT}}$ [49].

\section{Conclusion}

$\mathrm{AAN}_{\mathrm{mDOF}}$ robotic gait training was not superior to conventional gait training for improving $\mathrm{W}_{\mathrm{EXT}}$, spatiotemporal gait characteristics, functional gait tasks, or clinical scores in stroke survivors during their primary inpatient rehabilitation. However, we found some indication of a beneficial (kinematic) effect of $\mathrm{AAN}_{\mathrm{mDOF}}$ robotic gait training on peak knee flexion during the swing phase in a subgroup of participants with a predefined training goal aimed at improving foot clearance.

\section{Supplementary Information}

The online version contains supplementary material available at https://doi. org/10.1186/s12984-020-00800-4.

Additional file 1. Test statistics - External work, gait speed and secondary outcomes.

Additional file 2. Test statistics_-Individual training goals.

\section{Abbreviations}

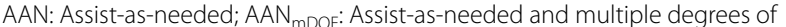
freedom; COM: Center of mass of the body; $E_{\mathrm{EXT}}$ : Energy level of the body; $W_{\text {EXT: }}$ : External mechanical work; FAC: Functional Ambulation Category; HADS: Hospital Anxiety and Depression Scale; MAS: Modified Ashworth Scale; MoCA: Montreal Cognitive Assessment; UCO: Utrechts Communicatie Onderzoek; IQR: Interquartile range.

\section{Acknowledgements}

The authors would like to thank Bart Nienhuis and MOOG for their technical support, and all therapists for their help in providing the gait training sessions.

\section{Authors' contributions}

$\mathrm{JA}$ and BF collected, analyzed and interpreted the data, and wrote the draft manuscript. BG, JR, VW, EA, AG and JB made substantial contributions to the experimental design, data interpretation and revision of the manuscript. All authors read and approved the final manuscript.

\section{Funding}

The authors disclosed receipt of the following financial support for the research, authorship and/or publication of this article: This work was supported by a grant [Grant Number 95104009] from the Netherlands 
Organization for Health Research and Development (ZonMw) and the Dutch Brain Foundation (Hersenstichting Nederland).

\section{Availability of data and materials}

The datasets used during the current study are available from the corresponding author on reasonable request.

\section{Ethics approval and consent to participate}

The study was designed following the Declaration of Helsinki. The study protocol (NL 50748.044.14) was approved by the Medical Ethical Committee Twente (Enschede, the Netherlands). All participants gave written informed consent.

\section{Consent for publication}

Not applicable.

\section{Competing interests}

None of the involved authors have any competing interests to report.

\section{Author details}

${ }^{1}$ Sint Maartenskliniek Research, PO Box 9011, 6500 GM Nijmegen, The Netherlands. ${ }^{2}$ Donders Institute for Brain, Cognition and Behaviour, Department of Rehabilitation, Radboud University Medical Center, Nijmegen, The Netherlands. ${ }^{3}$ Roessingh Research and Development, Enschede, The Netherlands. ${ }^{4}$ Roessingh Center for Rehabilitation, Enschede, The Netherlands. ${ }^{5}$ Department of Biomechanical Engineering, University of Twente, Enschede, The Netherlands. ${ }^{6}$ Department of Biomedical Signals and Systems, University of Twente, Enschede, The Netherlands.

Received: 9 September 2020 Accepted: 9 December 2020 Published online: 05 February 2021

\section{References}

1. Bohannon RW, Andrews AW, Smith MB. Rehabilitation goals of patients with hemiplegia. Int J Rehabil Res. 1988;11(2):181-4.

2. Harris JE, Eng JJ. Goal priorities identified through client-centred measurement in individuals with chronic stroke. Physiother Can. 2004;56(3):171-6.

3. Mumma CM. Perceived losses following stroke. Rehabil Nurs. 1986;11(3):19-24.

4. Balaban B, Tok F. Gait disturbances in patients with stroke. PM \& R. 2014;6(7):635-42.

5. Chen G, Patten C, Kothari DH, Zajac FE. Gait differences between individuals with post-stroke hemiparesis and non-disabled controls at matched speeds. Gait Posture. 2005;22(1):51-6.

6. Moseley A, Wales A, Herbert R, Schurr K, Moore S. Observation and analysis of hemiplegic gait: stance phase. Aust J Physiother. 1993;39(4):259-67.

7. Patterson KK, Parafianowicz I, Danells CJ, Closson V, Verrier MC, Staines WR, et al. Gait asymmetry in community-ambulating stroke survivors. Arch Phys Med Rehabil. 2008;89(2):304-10.

8. Olney SJ, Richards C. Hemiparetic gait following stroke. Part I: Characteristics. Gait Posture. 1996;4(2):136-48.

9. van der Kooi E, Schiemanck SK, Nollet F, Kwakkel G, Meijer JW, van de Port I. Falls are associated with lower self-reported functional status in patients after stroke. Arch Phys Med Rehabil. 2017;98(12):2393-8.

10. Durcan S, Flavin E, Horgan F. Factors associated with community ambulation in chronic stroke. Disabil Rehabil. 2016;38(3):245-9.

11. Chang WH, Kim MS, Huh JP, Lee PK, Kim YH. Effects of robot-assisted gait training on cardiopulmonary fitness in subacute stroke patients: a randomized controlled study. Neurorehabil Neural Repair. 2012;26(4):318-24.

12. Chua J, Culpan J, Menon E. Efficacy of an electromechanical gait trainer poststroke in Singapore: a randomized controlled trial. Arch Phys Med Rehabil. 2016;97(5):683-90.

13. Han EY, Im SH, Kim BR, Seo MJ, Kim MO. Robot-assisted gait training improves brachial-ankle pulse wave velocity and peak aerobic capacity in subacute stroke patients with totally dependent ambulation: Randomized controlled trial. Medicine. 2016;95(41):e5078.
14. Husemann B, Muller F, Krewer C, Heller S, Koenig E. Effects of locomotion training with assistance of a robot-driven gait orthosis in hemiparetic patients after stroke: a randomized controlled pilot study. Stroke. 2007;38(2):349-54.

15. Morone G, Bragoni M, losa M, De Angelis D, Venturiero V, Coiro P, et al Who may benefit from robotic-assisted gait training? A randomized clinical trial in patients with subacute stroke. Neurorehabil Neural Repair. 2011;25(7):636-44.

16. Ochi M, Wada F, Saeki S, Hachisuka K. Gait training in subacute nonambulatory stroke patients using a full weight-bearing gait-assistance robot: A prospective, randomized, open, blinded-endpoint trial. J Neurol Sci. 2015;353(1-2):130-6.

17. van Nunen MP, Gerrits KH, Konijnenbelt M, Janssen TW, de Haan A. Recovery of walking ability using a robotic device in subacute stroke patients: a randomized controlled study. Disabil Rehab Assist Technol. 2015;10(2):141-8.

18. Hidler J, Nichols D, Pelliccio M, Brady K, Campbell DD, Kahn JH, et al. Multicenter randomized clinical trial evaluating the effectiveness of the Lokomat in subacute stroke. Neurorehabil Neural Repair. 2009;23(1):5-13.

19. Emken JL, Benitez R, Reinkensmeyer DJ. Human-robot cooperative movement training: learning a novel sensory motor transformation during walking with robotic assistance-as-needed. J Neuroeng Rehabil. 2007;4:8.

20. Meuleman J, van Asseldonk E, van Oort G, Rietman H, van der Kooij H. LOPES II-design and evaluation of an admittance controlled gait training robot with shadow-leg approach. IEEE Trans Neural Syst Rehabil Eng. 2016;24(3):352-63.

21. Aurich-Schuler T, Grob F, van Hedel HJA, Labruyere R. Can Lokomat therapy with children and adolescents be improved? An adaptive clinical pilot trial comparing Guidance force, Path control, and FreeD. J Neuroeng Rehabil. 2017;14:14

22. Duschau-Wicke A, Caprez A, Riener R. Patient-cooperative control increases active participation of individuals with $\mathrm{SCl}$ during robot-aided gait training. J Neuroeng Rehabil. 2010;7:43.

23. Duschau-Wicke A, von Zitzewitz J, Caprez A, Lunenburger L, Riener R. Path control: a method for patient-cooperative robot-aided gait rehabilitation. IEEE Trans Neural Syst Rehabil Eng. 2010;18(1):38-48.

24. Veneman JF, Menger J, van Asseldonk EH, van der Helm FC, van der Kooij $\mathrm{H}$. Fixating the pelvis in the horizontal plane affects gait characteristics. Gait Posture. 2008;28(1):157-63.

25. Hidler J, Sainburg R. Role of Robotics in Neurorehabilitation. Top Spinal Cord Inj Rehabil. 2011;17(1):42-9.

26. Willems PA, Cavagna GA, Heglund NC. External, internal and total work in human locomotion. J Exp Biol. 1995;198(Pt 2):379-93.

27. Saunders JB, Inman VT, Eberhart HD. The major determinants in normal and pathological gait. J Bone Joint Surg Am. 1953;35(3):543-58.

28. de Carmo AA, Kleiner AF, Barros RM. Alteration in the center of mass trajectory of patients after stroke. Top Stroke Rehabil. 2015;22(5):349-56.

29. Detrembleur C, Dierick F, Stoquart G, Chantraine F, Lejeune T. Energy cost, mechanical work, and efficiency of hemiparetic walking. Gait Posture. 2003;18(2):47-55.

30. Balbinot G. Walking at non-constant speeds: mechanical work, pendular transduction, and energy congruity. Scand J Med Sci Sports. 2017:27(5):482-91.

31. Cavagna GA, Thys $H$, Zamboni A. The sources of external work in level walking and running. J Physiol. 1976;262(3):639-57.

32. Gage JR. Gait analysis. An essential tool in the treatment of cerebral palsy. Clin Orthop Relat Res. 1993;288:126-34.

33. Fugl-Meyer AR, Jaasko L, Leyman I, Olsson S, Steglind S. The post-stroke hemiplegic patient 1 a method for evaluation of physical performance. Scand J Rehabil Med. 1975;7(1):13-31.

34. Demeurisse G, Demol O, Robaye E. Motor evaluation in vascular hemiplegia. Eur Neurol. 1980;19(6):382-9.

35. Nasreddine ZS, Phillips NA, Bedirian V, Charbonneau S, Whitehead V, Collin I, et al. The Montreal Cognitive Assessment, MoCA: a brief screening tool for mild cognitive impairment. J Am Geriatr Soc. 2005;53(4):695-9.

36. Pijfers EMdVLA, Messing-Petersen $\mathrm{H}$. Het Utrechts Communicatie Onderzoek. Westervoort: Stichting Afasie Nederland; 1985. 
37. Veerbeek JM, van Wegen $E$, van Peppen $R$, van der Wees PJ, Hendriks E, Rietberg $\mathrm{M}$, et al. What is the evidence for physical therapy poststroke? A systematic review and meta-analysis. PLoS ONE. 2014;9(2):e87987.

38. Zeni JA Jr, Richards JG, Higginson JS. Two simple methods for determining gait events during treadmill and overground walking using kinematic data. Gait Posture. 2008;27(4):710-4.

39. Guyatt GH, Sullivan MJ, Thompson PJ, Fallen EL, Pugsley SO, Taylor DW et al. The 6-minute walk: a new measure of exercise capacity in patients with chronic heart failure. Can Med Assoc J. 1985;132(8):919-23.

40. Collen FM, Wade DT, Bradshaw CM. Mobility after stroke: reliability of measures of impairment and disability. Int Disabil Stud. 1990;12(1):6-9.

41. Podsiadlo D, Richardson S. The timed "Up \& Go": a test of basic functional mobility for frail elderly persons. J Am Geriatr Soc. 1991;39(2):142-8.

42. Wrisley DM, Marchetti GF, Kuharsky DK, Whitney SL. Reliability, internal consistency, and validity of data obtained with the functional gait assessment. Phys Ther. 2004;84(10):906-18.

43. Stoquart $G$, Detrembleur $C$, Lejeune TM. The reasons why stroke patients expend so much energy to walk slowly. Gait Posture. 2012;36(3):409-13.

44. Srivastava S, Kao PC, Kim SH, Stegall P, Zanotto D, Higginson JS, et al. Assist-as-needed robot-aided gait training improves walking function in individuals following stroke. IEEE Trans Neural Syst Rehabil Eng 2015;23(6):956-63.
45. Srivastava S, Kao PC, Reisman DS, Scholz JP, Agrawal SK, Higginson JS. Robotic assist-as-needed as an alternative to therapist-assisted gait rehabilitation. Int J Phys Med Rehabil. 2016;4:5.

46. Seo JS, Yang HS, Jung S, Kang CS, Jang S, Kim DH. Effect of reducing assistance during robot-assisted gait training on step length asymmetry in patients with hemiplegic stroke: a randomized controlled pilot trial. Medicine. 2018:97(33):e11792.

47. Mehrholz J, Thomas S, Werner C, Kugler J, Pohl M, Elsner B. Electromechanical-assisted training for walking after stroke. Cochrane Database Syst Rev. 2017;5:006185

48. Cavagna GA. Force platforms as ergometers. J Appl Physiol. 1975;39(1):174-9.

49. Pavei G, Seminati E, Cazzola D, Minetti AE. On the estimation accuracy of the 3D body center of mass trajectory during human locomotion: inverse vs forward dynamics. Front Physiol. 2017;8:129.

\section{Publisher's Note}

Springer Nature remains neutral with regard to jurisdictional claims in published maps and institutional affiliations.
Ready to submit your research? Choose BMC and benefit from:

- fast, convenient online submission

- thorough peer review by experienced researchers in your field

- rapid publication on acceptance

- support for research data, including large and complex data types

- gold Open Access which fosters wider collaboration and increased citations

- maximum visibility for your research: over 100M website views per year

At BMC, research is always in progress.

Learn more biomedcentral.com/submissions 